\title{
De-Speckling by Hybridization of Wavelet Transform and Bilateral Filter in Ultrasound Images
}

\author{
Parminder Pal Kaur \\ Yadwindra College of Engg., \\ Talwandi Sabo
}

\author{
Tejinderpal Singh \\ Yadwindra College of Engg., \\ Talwandi Sabo
}

\begin{abstract}
In this paper we introduce a de-noising technique with hybridization of wavelet transform and bilateral filter. In digital image processing, image is corrupted by various types of noise. But medical images are usually corrupted by speckle noise. So to remove noise, image de-speckling is very essential exercise of diagnose. To speckle noise reduction, multiplicative noise is converted into additive noise. Various filters are implemented for the reduction of speckle noise from ultrasound images. The proposed method uses wavelet transform and bilateral filter to reduce speckle noise from ultrasound images with homomorphic approach. We compare our results with mean filter, median filter, Gaussian filter, bilateral filter, wiener filter, Lee filter, Kuan filter and SRAD filters. So, we use bilateral filter using homomorphic approach with wavelet thresholding. The use of Weiner filter and soft thresholding provides better results to reduce speckle noise and gives better image quality with fine details. Another method is developed for mixed noise reduction from ultrasound images. We compare the results of proposed methods with the existing approaches in terms of PSNR and MSE. The hybridization of wavelet transforms and bilateral filter has been shown work well than other smoothing filters and mixed noise reduction methods.
\end{abstract}

\section{Keywords}

Bilateral filter, wavelet transform, PSNR

\section{INTRODUCTION}

Image processing is ubiquitous, with applications ranging from television to tomography, from photography to printing, from robotics to remote sensing. Analysis of images usually refers to processing of images by computer with the goal of finding what objects are presented in the image. Visual information plays an important role in almost all areas of our life. Today, much of this information is represented and processed digitally. Noise is the undesired information that affects the quality of image. There is different sources of noise that may contaminate any digital image and degrade the quality. The overall characteristics of noise in an image depend on many factors, namely sensor type, dimensions of pixel, temperature, exposure time, and speed of the sensor. Image de-noising refers to the recovery of a digital image that has been contaminated by noise. Image de-noising is used to

reduce the noise while retaining the important features of signal. The purpose of reducing the noise from an image is to estimate the original image from the noisy data. Low image quality is an obstacle for effective extraction of features, analysis, recognition and quantitative measurements. Therefore, there is a fundamental need of noise reduction from medical images [11]. Ultrasound imaging is considered noninvasive that is practically harmless to the human body, portable, accurate and cost effective. This technique is used to visualize muscles and many internal organs, their size, structure and any pathological injuries. These features have made the ultrasound imaging the most prevalent diagnostic tool in nearly all hospital in the world. The quality of ultrasound images is limited due to some factors which originate physical phenomena and imperfections of imaging system design. This technique is inexpensive and portable than other imaging techniques such as magnetic resonance imaging MRI and computed tomography CT.

Feature-preserved de-noising is of great interest in medical image processing. Ultrasound imaging is widely used in the medical field. Ultrasound is considered to be one of the most powerful techniques for imaging organs and soft tissue structures in the body of human. The use of ultrasound images is that these are less expensive, no harm to human body. Ultrasound images are also suffered from one noise that is speckle noise. Speckle noise is one of the major sources of noise. Speckle noise is an inherent property of medical ultrasound imaging, and it generally tends to reduce the resolution of image and contrast, delectability of objects, thereby reducing the diagnostic value of this imaging modality. It has negative impact on medical imaging [12]. As the noise removal causes blurring of the image, so image denoising still remains the challenge for researchers. Noise removal methods also reduce some useful information from the image which makes the image unusable for feature extraction and analysis [10].

There are two types of noise additive and multiplicative. The additive noise is an unwanted signal that gets added into some relevant signal. Multiplicative noise refers to an unwanted signal that gets multiplied into some relevant signal during capture, transmission, or other processing. Multiplicative noise is difficult to reduce as compared to additive noise [10].Noise estimation methods in wavelet domain are most popular among the effective de-noising procedures. Also, different type of filtering is used to reduce the noise from images. Filters used are of two types, that is, linear filtering and non-linear filtering.

\section{MATERIALS AND METHODS}

\subsection{Performance measurements in Ultrasound images}

Peak signal to noise ratio (PSNR): PSNR is the peak signalto-noise ratio in decibels $(\mathrm{dB})$. The PSNR is only meaningful for data encoded in terms of bits per sample, or bits per pixel. For example, an image with 8 bits per pixel contains integers from 0 to 255. PSNR is computed as follows:-

$$
P S N R=10 \log _{10}\left[\frac{255 \times 255}{M S E}\right]
$$


MSE (Mean Squared error): MSE is the mean square error between a signal $f(x y)$ and an approximation $f^{\prime \prime}(x y)$ is the squared norm of the difference divided by the number of elements in the signal.

$$
M S E=\sum_{M, N} \frac{(g(m, n)-f(m, n))^{2}}{M \times N}
$$

In this method, speckle noise is added into an ultrasound image and then homomorphic approach is applied to an image with bilateral filter. The image is decomposed with one level wavelet transform, which divide the image into approximation and detail sub-bands. On detail sub-bands wiener filtering approach is applied to provide better results. Then the approximation band is again decomposed into second level approximation and detail sub-bands $\{\mathrm{AA}, \mathrm{HH}, \mathrm{VV}, \mathrm{DD}\}$. After $2^{\text {nd }}$ level, wavelet thresholding (soft thresholding) technique is applied for reduction of noise. On modified wavelet coefficient inverse wavelet transform is applied two times. To get de-speckled image, bilateral filter is applied on inverse coefficient, which gives better image quality with reduce speckle noise.

The details of the wavelet decomposition and bilateral filter are as follows:

\subsection{Wavelet Decomposition}

The discrete wavelet transform (DWT) of an image of the size $M \times N$ pixels produces one 2D scaling function $\varphi(x, y)$ and three 2D wavelet functions $\psi^{H}(x, y), \psi^{V}(x, y), \psi^{D}(x, y)$. The two dimensional scaling and translation functions can be defined as follows:

$$
\begin{array}{r}
\varphi_{j, m, n}(x, y)=2^{j / 2} \varphi\left(2^{j} x-m, 2^{j} y-n\right) \\
\psi_{j, m, n}^{i}(x, y)=2^{j / 2} \psi^{i}\left(2^{j} x-m, 2^{j} y-n\right), \quad i=\{H, V, D\}
\end{array}
$$

where $j$ represent scale, $m$ and $n$ represent shift along the horizontal and vertical directions, and index $i$ identifies the directional wavelets which are horizontal $(H)$, vertical $(V)$, and diagonal $(D)$. The DWT of an image of the size $M \times N$ pixels can be expressed as:

$$
\begin{aligned}
& W_{\varphi}\left(j_{0}, m, n\right)=\frac{1}{\sqrt{M N}} \sum_{x=0}^{M-1} \sum_{y=0}^{N-1} f(x, y) \varphi_{\mathrm{j}_{0}, m, n}(x, y) \\
& W_{\psi}^{i}(j, m, n)=\frac{1}{\sqrt{M N}} \sum_{x=0}^{M-1} \sum_{y=0}^{N-1} f(x, y) \psi_{\mathrm{j}, m, n}^{i}(x, y) i=
\end{aligned}
$$$$
\{H, V, D\}
$$

where $W_{\varphi}\left(j_{0}, m, n\right)$ coefficients define as an approximation of $f(x, y)$ at scale $j_{0}$. The $W_{\psi}^{i}(j, m, n)$ coefficients add details for scale $j \geq j_{0}$ for the above mentioned directions ( $H, V$, and $D$ ).

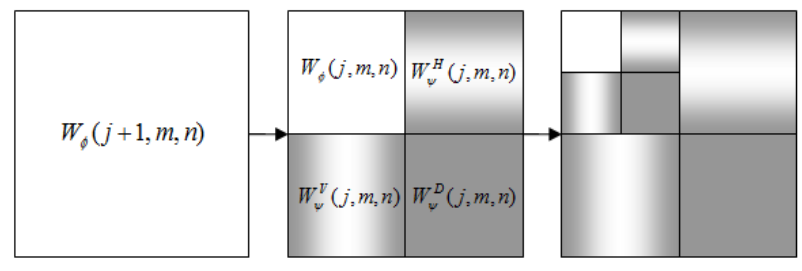

Fig 1: 2-DWT Decomposition

\subsection{Soft Thresholding}

The most frequently used technique for Ultrasound image noise reduction using the wavelet coefficients is thresholding. It is assumed that the wavelet coefficients with values lower than a particular threshold value $T$ corresponds to noisy samples and they can be therefore cancelled, which leads to noise reduction in the image domain. When the remaining coefficients are unaffected, it is called hard thresholding:

- Compute the forward transform of the image to be denoised and decompose the image into sub bands.

- Compute the threshold from the first scale HH (vertical details) band using the MAD (median absolute deviation) using the following formula considering that most of the noise is present in that band.

$\sigma(\operatorname{mad})=\operatorname{median}\{|w j|: j=1,2, \ldots . k / 2\} / .6745$

Apply the shrinkage step (modifying the wavelet coefficients in the sub bands) using the following shrinkage rules.

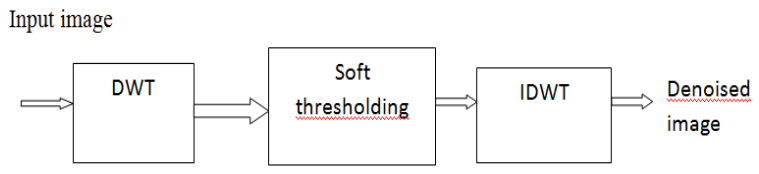

Fig 2: De-noising procedure with multi-scale transform

\subsection{Bilateral filter}

It is convolution filter. A bilateral filter is a combination of two filters, working both on the area and range of the function (image). The idea underlying bilateral filtering is to do in the range of an image what traditional filters do in its domain. Two pixels can be close to one another, that is, they occupy nearby spatial location, or they can be similar to one another, that is, have nearby values, possibly in a perceptually meaningful fashion. Closeness refers to vicinity in the domain, similarity to vicinity in the range. Traditional filtering is domain filtering and enforces closeness by weighing pixel values with coefficients that fall off with distance. Similarly, we define a range filtering, which averages image values with weights that decay with dissimilarity. Range filters are nonlinear because their weights depend on image intensity or color. Most important is that they preserve edges. In this approach, both range and domain filters are combined. The main reason of taking this filter is that it only smooth regions but does not smooth edges. Bilateral filter smooth image but preserve edges. It operates both in domain and range of the image. Bilateral filter gives better performance when we are taking log of it because it is use for additive noise [6]. The algorithmic idea of bilateral filter is it smooth as usual in the domain of the image e.g. Gaussian and it does not smooth when pixels are not similar e.g. edges.

The bilateral filter is defined as follows:-

$$
y[i, j]=\frac{\sum_{(s, t) \epsilon N(i, j)} W d[s, t] W r[s, t] g[s, t]}{\sum_{(s, t) \in N(k, l)} W d[s, t] W r[s, t]}
$$


Where $\mathrm{Wd}$ and $\mathrm{Wr}$ are the domain and range weights, which are described as in Equation (9) and (10):

$$
\begin{aligned}
& \mathrm{Wd}(\mathrm{s}, \mathrm{t})=\exp \left\{-\frac{([i-s]+[j+t])^{2}}{2 \sigma_{d}^{2}}\right\} \\
& \mathrm{Wr}(\mathrm{s}, \mathrm{t})=\exp \left\{-\frac{(g(i, j)-g[s, t])^{2}}{2 \sigma_{r}^{2}}\right\}
\end{aligned}
$$

The domain and range parameters $\sigma d$ and $\sigma r$ control the behavior of weights.

The bilateral filter is used in the proposed algorithm since it is non iterative and simple. On noisy image the homomorphic approach is applied with bilateral filter to provide better performance. When filtering noise using a Gaussian filter there is a problem of edge blurring between areas of different colors.

\section{PROPOSED METHOD}

\subsection{Algorithm of speckle noise reduction}

Step 1: The speckle noise is added in the ultrasound image.

Step 2: After adding the speckle noise in an image, homomorphic approach (logarithm) is applied on an image.

Step 3: Bilateral filter is applied after the logarithm of an image. After applying the Bilateral filter, exponential operation is performed of an image for inverse logarithm.

Step 4: The image is decomposed to one level using discrete wavelet transform, which gives rise to four sub-bands (approximation sub-band LL1, detail sub-bands LH1, HL1 and $\mathrm{HH} 1$ ).

Step 5: Wiener smoothing filter is applied on the detailed subbands.

Step 6: Discrete wavelet transform is again applied on approximation sub-band LL1 which again gives rise to four sub-bands (approximation sub-band LL2, detail sub-bands LH2, HL2 and HH2).

Step 7: The wavelet shrinkage technique soft thresholding is applied on the detail sub-band coefficients of second level.

Step 8: The inverse wavelet transform is applied two times on the modified wavelet coefficients.

Step 9: The bilateral filter is again applied at the last stage with homomorphic approach to get the de-speckled image.

Two parameters PSNR and MSE are considered for comparing the results of the proposed method with the existing methods in the literature.

\section{EXPERIMENTAL RESULTS}

The proposed method is implemented in MATLAB R2010a with Microsoft windows environment on a PC with $3.0 \mathrm{GHz}$ CPU \& 3GB RAM. In our experiment we have chosen a grayscale image of size $352 \times 256$ pixel. The algorithm of the proposed method is given above. The ultrasound image is given in Fig.3. The results of speckle noise reduction of proposed method are shown in Table 1 and in Figs. 4 to 12.
Table 1. Experimental results of proposed method

\begin{tabular}{|c|c|c|c|c|}
\hline \multirow{2}{*}{$\begin{array}{l}\text { Speckle } \\
\text { noise } \\
\text { variance }\end{array}$} & \multicolumn{2}{|c|}{ Noisy image } & \multicolumn{2}{c|}{ Proposed method } \\
\cline { 2 - 5 } & PSNR & MSE & PSNR & MSE \\
\hline 0.01 & 28.3552 & 94.9646 & 37.985 & 10.3415 \\
\hline 0.02 & 25.34 & 190.142 & 35.2391 & 19.4612 \\
\hline 0.03 & 23.5742 & 285.538 & 32.6172 & 35.5927 \\
\hline 0.04 & 22.3405 & 379.34 & 30.6967 & 55.3873 \\
\hline 0.05 & 21.3693 & 474.41 & 29.0697 & 80.5579 \\
\hline 0.06 & 20.5709 & 570.155 & 27.7612 & 108.883 \\
\hline 0.07 & 19.8977 & 665.744 & 26.776 & 136.609 \\
\hline 0.08 & 19.3188 & 760.683 & 25.8861 & 167.674 \\
\hline 0.09 & 18.8143 & 854.384 & 25.1742 & 197.543 \\
\hline
\end{tabular}

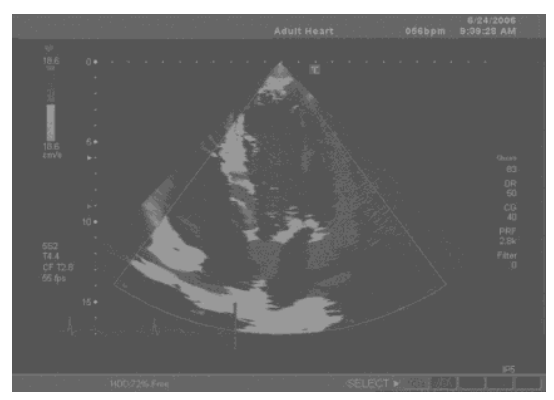

Fig 3: Original Image

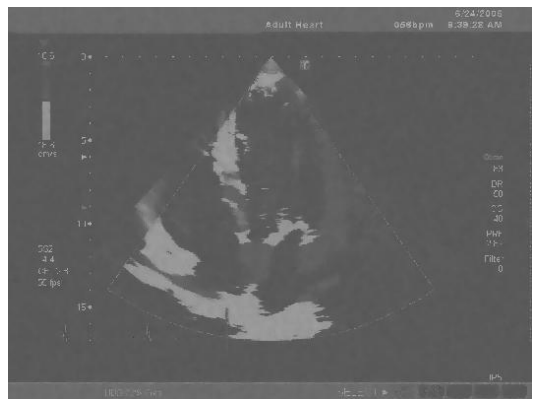

Fig 4: Result of proposed method on the image with speckle noise variance 0.01

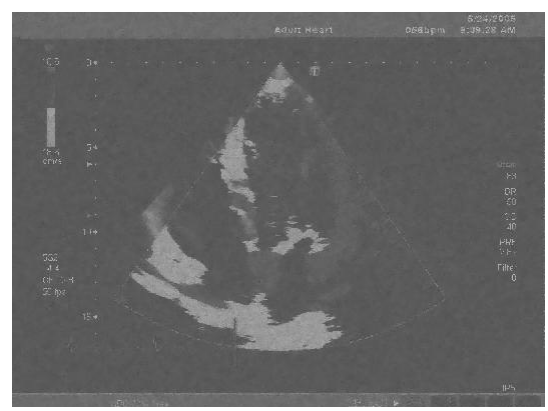

Fig 5: Result of proposed method on the image with speckle noise variance 0.02 


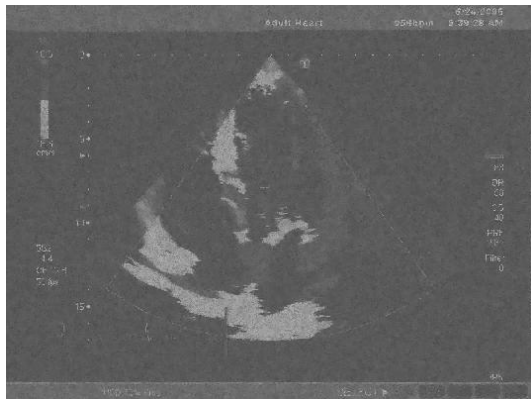

Fig 6: Result of proposed method on the image with speckle noise variance 0.03

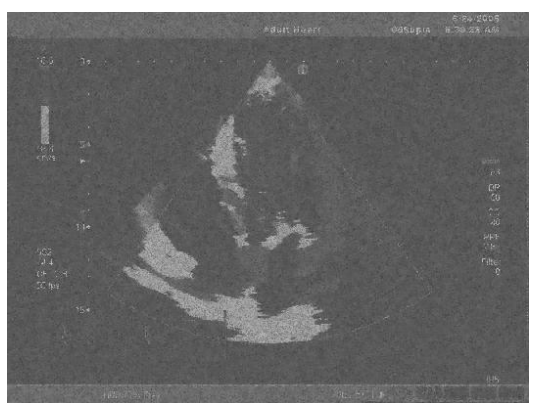

Fig 7: Result of proposed method on the image with speckle noise variance 0.04

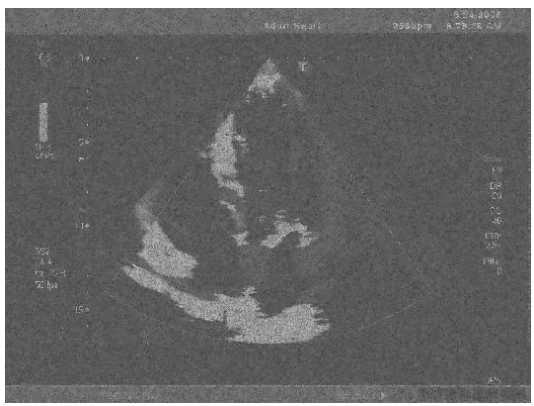

Fig 8: Result of proposed method on the image with speckle noise variance 0.05

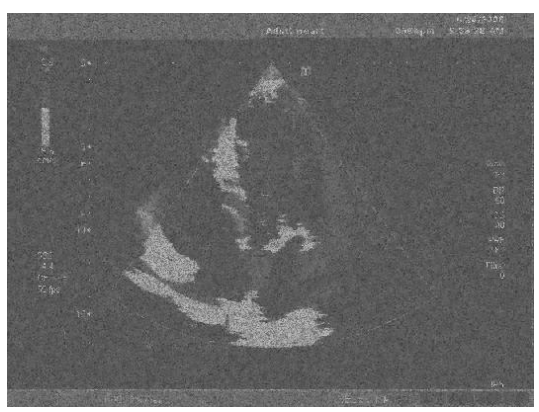

Fig 9: Result of proposed method on the image with speckle noise variance 0.06

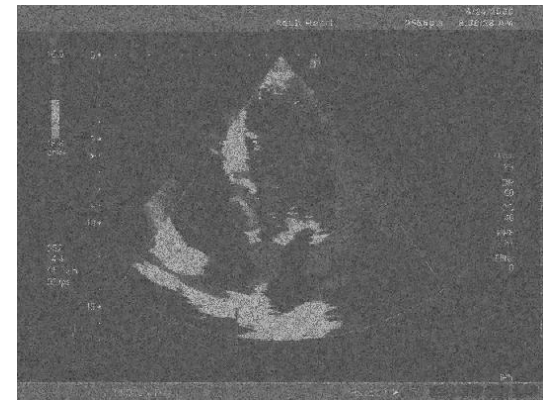

Fig 10: Result of proposed method on the image with speckle noise variance 0.07

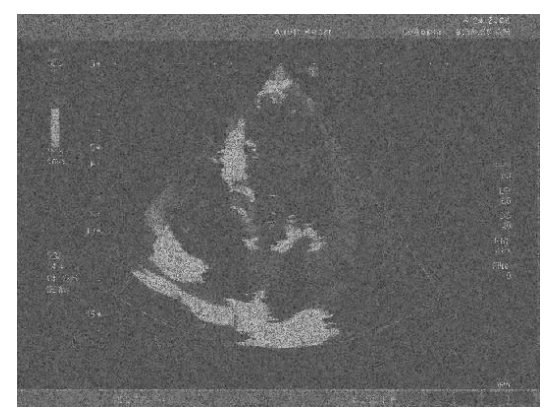

Fig 11: Result of proposed method on the image with speckle noise variance 0.08

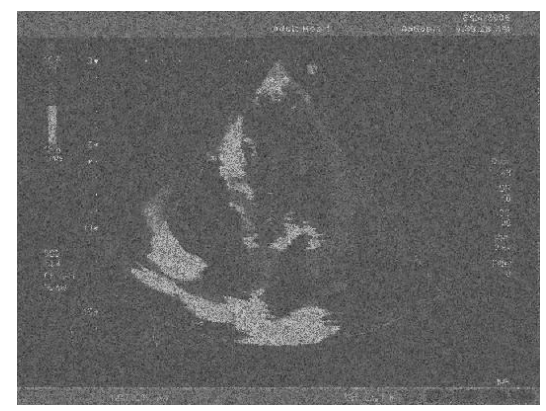

Fig 12: Result of proposed method on the image with speckle noise variance 0.09

The proposed techniques are compared with each other and observed that the proposed algorithm for mixed noise reduction is giving very good results for low level of Gaussian noise. However, proposed algorithm for speckle noise reduction is giving better results for all level of speckle noises. The proposed technique for speckle noise reduction is also compared with other filters available in the literature and results are compared in Table 2. It is observed from the table that proposed method is giving better results as compared to other techniques. 
Table 2. Comparison of proposed method with existing methods in terms of PSNR

\begin{tabular}{|c|c|c|c|c|c|c|c|c|c|}
\hline $\begin{array}{l}\text { Noise } \\
\text { variance }\end{array}$ & 0.01 & 0.02 & 0.03 & 0.04 & 0.05 & 0.06 & 0.07 & 0.08 & 0.09 \\
\hline Mean filter & 32.1175 & 31.1121 & 30.2732 & 29.6158 & 28.9911 & 28.4499 & 28.0083 & 27.5911 & 27.1997 \\
\hline Median & 31.0746 & 29.3047 & 28.0412 & 27.0965 & 26.2876 & 25.5963 & 25.0293 & 24.5085 & 24.0406 \\
\hline Bilateral & 31.7587 & 28.9785 & 27.2764 & 26.0887 & 25.1329 & 24.3415 & 23.6842 & 23.1143 & 22.6158 \\
\hline Gaussian & 32.9318 & 32.7104 & 32.2444 & 31.6405 & 30.8287 & 29.9225 & 29.0421 & 28.1287 & 27.2188 \\
\hline Weiner & 35.0689 & 32.2416 & 30.5285 & 29.3665 & 28.4152 & 27.6247 & 27.0228 & 26.4419 & 25.9612 \\
\hline Lee & 32.8286 & 31.6583 & 30.7177 & 29.9871 & 29.3142 & 28.7254 & 28.251 & 27.8094 & 27.3985 \\
\hline Kuan. & 32.3634 & 32.0677 & 31.7532 & 31.5575 & 31.2793 & 31.0273 & 30.8208 & 30.5434 & 30.0462 \\
\hline SRAD & 32.4695 & 31.8471 & 31.2841 & 30.7506 & 30.223 & 29.6541 & 29.0807 & 28.3732 & 27.663 \\
\hline $\begin{array}{l}\text { Soft } \\
\text { thresholding }\end{array}$ & 28.4549 & 25.3999 & 23.6208 & 22.3864 & 21.4073 & 20.6052 & 19.9303 & 19.3557 & 18.8465 \\
\hline $\begin{array}{l}\text { Hard } \\
\text { thresholding }\end{array}$ & 30.6848 & 27.4346 & 25.4903 & 24.2202 & 23.1853 & 22.3292 & 21.648 & 21.1222 & 20.4978 \\
\hline $\begin{array}{l}\text { Proposed } \\
\text { method }\end{array}$ & 37.985 & 35.2391 & 32.6172 & 30.6967 & 29.0697 & 27.7612 & 26.776 & 25.8861 & 25.1742 \\
\hline
\end{tabular}

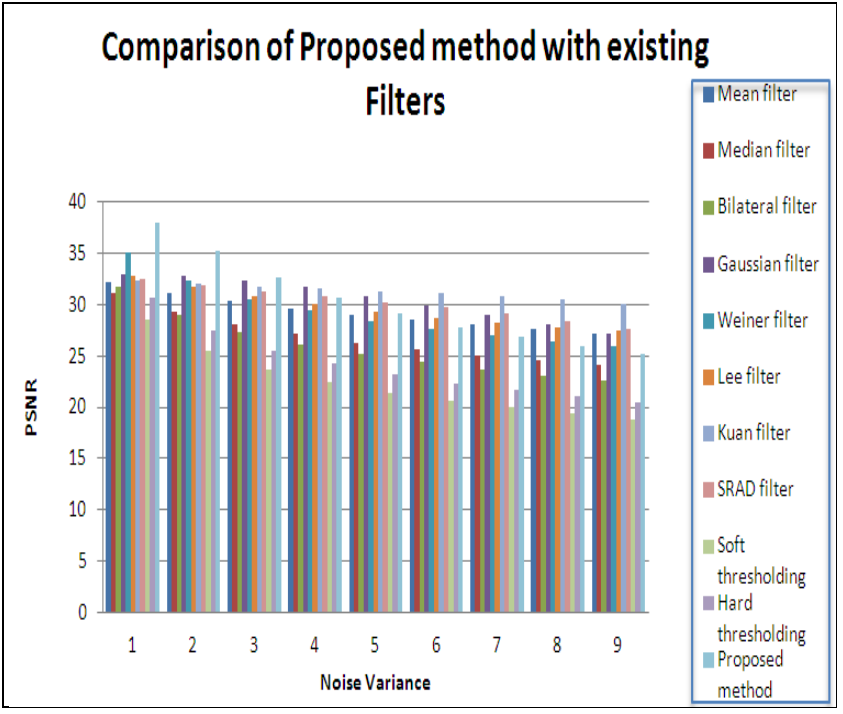

Fig 13: Graphical representation of the comparison $b / w$ proposed method and existing filters

\section{CONCLUSION AND FUTURE WORK}

In this paper, various previous methods are implemented for speckle noise reduction like Mean, Median, Gaussian and Bilateral, wiener, Lee, Kuan and SRAD filtering. The major objective of this research is to de-speckling the ultrasound images. We propose technique for de-speckling using wavelets transform and bilateral filter in ultrasound images to reduce speckle noise, which gives better results as compared to other techniques. The different performance analyses are experimented on ultrasound images with filtering methods. The performance analyses are calculated by PSNR and MSE values. The proposed method compares with other smoothing filtering techniques which gives better performance in terms of PSNR and MSE. In this work we have also implemented mixed noise method on ultrasound images to reduce speckle and Gaussian noise and compare speckle noise method with mixed noise method. According to their results proposed method is giving better performance in terms of PSNR and MSE than previous implemented work. The results show that bilateral filter and soft thresholding gives better results and performance than others filter. So the future work is to get more optimum result with wavelet transform and bilateral filter for reducing speckle noise by preserving edges and getting more useful information of ultrasound images.

As a result of these experiments in the previous study, we can reach the following Conclusions:

1. Clearly, the homomorphic approach with bilateral filter, the strong noise is eliminated most effectively.

2. For the gray-scale images, wavelet thresholding like soft thresholding will still keep some random noises in the texture which can be clearly observed, while again use of bilateral filter can reduce the noises in the texture very well.

3. Bilateral filter can use any type of wavelet thresholding method to reduce mixed noise using same parameters; the proposed method has the best output performance.

In future work, there will be better optimal results of using wavelet transform and bilateral filter. We have tried to achieve the objectives envisioned at the beginning of the thesis through the contributions presented in the thesis, but the research is yet not complete. The proposed method for Despeckling by Hybridization of wavelet and bilateral filter in ultrasound images can be further improved in many ways and has enormous scope for expansion. Some of the directions for future work are:

1. This method can be improved by the implementation of any other approach at the first and second level of wavelet transform coefficients.

2. This method can also be used for MRI images for better results.

\section{REFERENCES}

[1] Haung, T. S.,Yang, G. J.,Tang, G. Y., 1979 A fast twodimensional median filtering algorithm, IEEE Trans. on Acoustics, Speech and Signal Processing.

[2] Loupas, T., Mcdicken, W. N., Allan, P. N., 1989. An adaptive weighted median filter for speckle suppression in medical ultrasonic images, IEEE Trans. on Circuit and System.

[3] Perona, P., Malik, J., 1990. Scale-space and edge detection using anisotropic diffusion, IEEE Trans on Pattern Analysis and Machine Intelligence.

[4] Donovo L David., 1995. De-noising by Soft Thresholding, IEEE Transaction on Information Theory.

[5] Tomasi, C., Manduchi, R., 1998. Bilateral filtering for gray and color images, In Proceedings of IEEE Sixth International Conference on Computer Vision, Bombay.

[6] Addison P S., 2002. The Illustrated Wavelet Transform Handbook, Institute of physics. 
[7] Stippel G., Philips W., Lemahieu I., Govaert P., 2002. A Medical Feature Enhancing New De-noising Technique for Ultrasound Images, Digital Image Computing Techniques and Applications, Melbourne, Australia.

[8] Chen, Y., Raheja, A., 2005. Wavelet lifting for speckle noise reduction in ultrasound images, In Proceedings of IEEE Twenty-Seventh Annual Conference on Engineering in Medicine and Biology, Shanghai, China.

[9] Khare, A., Tiwary, U. S., 2005. A new method for deblurring and denoising of medical images using complex wavelet transform In Proceedings of IEEE Twenty-Seventh Annual conference on Engineering in Medicine and Biology, Shanghai, China.

[10] Oleg V. Michailovich., Allen Tannenbaum., 2006. Despeckling of Medical Ultrasound Images, IEEE transactions on ultrasonics, ferroelectrics, and frequency control, january.

[11] Chunhong Zhao., Liu Mingyu., Ji Hongbing, 2007. A new speckle reduction method of medical ultrasonic image in International Conference on Bio informatics and Biomedical Engineering.

[12] Zhang Ming., Guntuk K Bahadir., 2008. Multiresolution bilateral filtering for image de-noising, IEEE Transactions on Image Processing.

[13] Xiaona Zhi., Tianfu Wang, 2008. An anisotropic diffusion filter for ultrasonic speckle reduction, IEEE Transactions on Image Processing.

[14] S.Sudha, G.R.Suresh and R.Sukanesh, 2009. Speckle Noise Reduction in Ultrasound Images by Wavelet Thresholding based on Weighted Variance, International Journal of Computer Theory and Engineering.
[15] Qiang, L. W., Chang, M. F., Mei, Z. Y., Lu, Y., 2010. An algorithm of noise reduction for ultrasonic image on time difference method based on an improved median filter.

[16] Kaur Amandeep., Singh Karamjeet., 2010. Speckle Noise Reduction by Using Wavelets, National Conference on Computational Instrumentation CSIO Chandigarh.

[17] F. Yousefi Rizi, H. Ahmed Naubori and S. K Satarehdan., 2011. Wavelet-Based ultrasound image denoising: Performance and Comparison, International conference of IEEE EMBS.

[18] Bartuseka K., Prinosil J. Smekal Z., 2011. Wavelet-based de-noising techniques in MRI", computer methods and programs in biomedicine.

[19] Samusarma J Saraswathi., Brinda Mary., 2011. Denoising of mixed noise in ultrasound images, International Journal of Computer Science.

[20] Roy S., Sinha N., Sen A. K., 2012. An Efficient Denoising Model based on Wavelet and Bilateral Filters, International Journal of Computer Applications.

[21] V Naga Prudhvi Raj., T Venkateswarlu, 2012. Ultrasound Medical Image Denoising using Hybrid Bilateral Filtering, International Journal of Computer Applications.

[22] Vanithamani, R., Umamaheswari, G., 2014.Speckle Reduction In Ultrasound Images Using Neighshrink And Bilateral Filtering, International Journal of Computer Science. 